\title{
Incoherent non-Fermi-liquid scattering in a Kondo lattice
}

\author{
JOHNPIERRE PAGLIONE*, T. A. SAYLES, P.-C. HO, J. R. JEFFRIES AND M. B. MAPLE \\ Department of Physics, University of California at San Diego, La Jolla, California 92093, USA \\ *e-mail: paglione@physics.ucsd.edu
}

One of the most notorious non-Fermi-liquid properties of both archetypal heavy-fermion systems ${ }^{1-4}$ and the high- $T_{c}$ copper oxide superconductors ${ }^{5}$ is an electrical resistivity that evolves linearly (rather than quadratically) with temperature, $T$. In the heavy-fermion superconductor $\mathrm{CeCoIn}_{5}$ (ref. 6), this linear behaviour was one of the first indications of the presence of a zero-temperature instability, or quantum critical point. Here, we report the observation of a unique control parameter of $T$-linear scattering in $\mathrm{CeCoIn}_{5}$, found through systematic chemical substitutions of both magnetic and non-magnetic rareearth, $R$, ions into the Ce sublattice. We find that the evolution of inelastic scattering in $\mathrm{Ce}_{1-x} \mathrm{R}_{x} \mathrm{CoIn}_{5}$ is strongly dependent on the $f$-electron configuration of the $\mathrm{R}$ ion, whereas two other key properties-Cooper-pair breaking and Kondo-lattice coherence-are not. Thus, T-linear resistivity in $\mathrm{CeCoIn}_{5}$ is intimately related to the nature of incoherent scattering centres in the Kondo lattice, which provides insight into the anomalous scattering rate synonymous with quantum criticality ${ }^{7}$.

Although recent theories ${ }^{4,8-10}$ provide possible routes to an explanation of $T$-linear resistivity-found in both $f$-electron systems (for example, $\mathrm{Y}_{1-x} \mathrm{U}_{x} \mathrm{Pd}_{3}$ (ref. 1), $\mathrm{CeCu}_{6-x} \mathrm{Au}_{x}$ (ref. 2), $\mathrm{YbRh}_{2} \mathrm{Si}_{2}$ (ref. 3) and $\mathrm{CeCu}_{2} \mathrm{Si}_{2}$ (ref. 4)) and the normal state of the cuprate superconductors ${ }^{5}$-a general interpretation awaits arrival ${ }^{7}$. Several paradoxical features regarding this anomalous scattering rate continue to defy understanding, such as its persistence over decades of energy scales ${ }^{1,3,5}$ and down to millikelvin temperatures in three-dimensional materials ${ }^{1-4,6}$, its coexistence with conventional $\left(T^{2}\right)$ Hall-angle scattering ${ }^{11,12}$ and its inconsistency with oneparameter scaling ${ }^{13}$. Most recently, its observation over three decades of $T$ at the field-tuned quantum critical point (QCP) of $\mathrm{CeCoIn}_{5}$ has been linked to a violation of the Wiedemann-Franz $\mathrm{law}^{14}$, an indication that this scattering rate is associated with the failure of Fermi-liquid theory in its most basic form.

Here, we present a rigorous study of the effects of rare-earth substitution on three closely related features of the exotic metal $\mathrm{CeCoIn}_{5}$ : unconventional superconductivity, Kondo-lattice coherence and anomalous charge-carrier scattering. By diluting the Ce lattice within high-quality single-crystal specimens of $\mathrm{Ce}_{1-x} \mathrm{R}_{x} \mathrm{CoIn}_{5}$ with both non-magnetic (full or empty $4 f$-shell) and stable- $4 f$-moment substituent ions of varying size and electronic configuration, we are able to inject both 'Kondo holes' (isoelectronic ions without magnetic moments) and strongly localized magnetic moments into the coherent Kondo lattice. This has allowed us to probe the spin exchange between the $\mathrm{Ce}^{3+}$ localized magnetic moments and the spins of the conduction electrons involved in Cooper pairing, Kondo screening and anomalous transport in a controlled way, revealing a surprising contrast between the response of coherent phenomena and non-Fermi-liquid behaviour to this perturbation.

Figure 1 shows the evolution of both the superconducting transition temperature $T_{\mathrm{c}}$ (identified by the transition in resistivity, $\rho$ ) and Kondo-lattice coherence temperature $T_{\text {coh }}$ (identified by the maximum in $\rho(T))$ for all rare-earth substitutions made in $\mathrm{Ce}_{1-x} \mathrm{R}_{x} \mathrm{CoIn}_{5}$ through the complete range of concentrations where both features exist. As shown, the salient features are the same for all variants: as a function of residual resistivity $\left(\rho_{0} \sim x\right.$ - see the Methods section), both $T_{c}$ and $T_{\text {coh }}$ are suppressed to zero temperature at rates irrespective of the nature of the rare-earth ion, which spans both magnetic $\left(\mathrm{Pr}^{3+}, \mathrm{Gd}^{3+}, \mathrm{Dy}^{3+}, \mathrm{Er}^{3+}\right)$ and non-magnetic $\left(\mathrm{Y}^{3+}, \mathrm{Yb}^{2+}, \mathrm{Lu}^{3+}\right) f$-electron configurations. This highlights the insensitivity of two 'coherent' electronic properties of $\mathrm{CeCoIn}_{5}$, heavy-fermion superconductivity and Kondo-lattice screening, to the magnetic configuration of the substituted rareearth ions, the implications of each we will consider in turn.

The pair-breaking effect in unconventional superconductors arises via both potential (non-magnetic) and spin-flip scattering mechanisms. Potential scattering was shown via La substitution in $\mathrm{CeCoIn}_{5}$ to follow the Abrikosov-Gor'kov (AG) model for an anisotropic order parameter ${ }^{15}$, where it is well known that superconductivity is destroyed once the mean free path, $l_{\mathrm{mfp}}$, approaches the superconducting coherence length, $\xi$. Here, we estimate this critical scattering length to be $l_{\mathrm{cr}} \simeq 180 \AA$ at the point where $T_{\mathrm{c}} \rightarrow 0$ (that is, at $\rho_{\mathrm{cr}} \simeq 20 \mu \Omega \mathrm{cm}$, Fig. 1 ), assuming that the proportionality between $l_{\mathrm{mfp}}(x=0) \simeq 1,200 \AA$ (ref. 16) and $\rho(x=0)$ near $T_{\mathrm{c}}$ is independent of doping. This value is roughly twice the in-plane coherence length $\xi_{a}=80 \AA$ (ref. 6) and consistent with previous work $^{15}$. Interestingly, the value $\rho_{\text {cr }} \simeq 20 \mu \Omega \mathrm{cm}$ coincides with that found in the series $\mathrm{CeCoIn}_{5-x} \mathrm{Sn}_{x}$ (ref. 17), where $\mathrm{Sn}$ substitution for In preferentially occurs in the Ce-In layers ${ }^{18}$. In the absence of any dependence on replacement-ion size, as demonstrated by the contrast in metallic radii of $\mathrm{Lu}$ $(1.735 \AA)$ and $\mathrm{Y}(1.801 \AA)$, pair-breaking in $\mathrm{CeCoIn}_{5}$ thus seems to be dominated by general disorder in the $\mathrm{CeIn}_{3}$ planes.

The spin-flip interaction imposed on Cooper pairs by magnetic impurities is characterized by a further pair-breaking term $\Delta T_{\mathrm{c}} \propto \mathcal{g}^{2} D_{J}$, which includes the exchange interaction parameter $g$ and the de Gennes factor $D_{J}=(g-1)^{2} J(J+1)$, with the latter reflecting the classic competition between superconductivity and magnetism $^{19}$. The absence of a dependence of $\Delta T_{c}$ on this term in $\mathrm{Ce}_{1-x} \mathrm{R}_{x} \mathrm{CoIn}_{5}$ is intriguing, but not unprecedented. In $\mathrm{UPt}_{3}$, the insensitivity of $\Delta T_{\mathrm{c}}$ to $D_{J}$ is attributable to an odd-parity pairing state, where an equal Zeeman shift on parallel spin states renders the spin-flip process ineffective ${ }^{20}$. In the spin-singlet cuprates, $T_{\mathrm{c}}$ is insensitive to the flavour of the rare-earth ion, $\mathrm{R}$, placed 


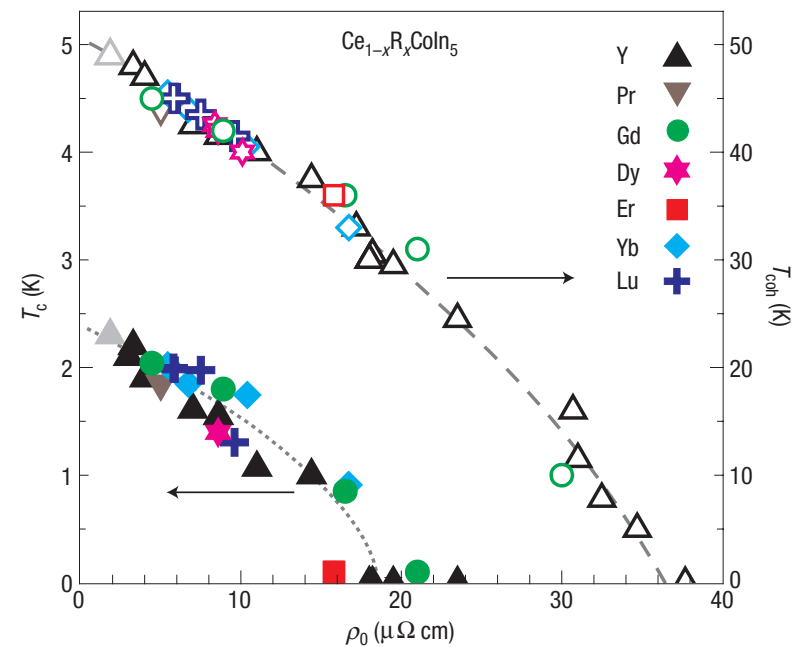

Figure 1 Dependence of superconducting transition temperature, $T_{\mathrm{c}}$, and Kondo-lattice coherence temperature, $\boldsymbol{T}_{\text {coh }}$ of $\mathrm{Ce}_{1-x} \mathrm{R}_{x} \mathrm{Coln}_{5}$ on rare-earth concentration. Plotted as a function of residual resistivity $\left(\rho_{0} \sim x\right.$ - see the Methods section), this figure highlights the absence of any effect of the electronic configuration of replacement-ion $\mathrm{R}$ on either $T_{\mathrm{c}}$ (filled symbols) or $T_{\text {coh }}$ (open symbols) as they evolve from $x=0$ (grey triangles). Note the lack of contrast between two particular species that are similar in all respects except $f$-electron filling: both $\mathrm{Y}^{3+}\left(5 s^{2} 4 d^{1}\right)$ and $\mathrm{Gd}^{3+}\left(6 s^{2} 4 f^{7} 5 d^{1}\right)$ are isovalent with $\mathrm{Ce}^{3+}$ $\left(6 s^{2} 4 f^{1} 5 d^{1}\right)$ and have nearly identical metallic radii of $1.801 \AA$, slightly smaller than that of $\mathrm{Ce}(1.825 \AA)$ and yielding a similarly small $(\sim 1 \%)$ change of the lattice parameters on substitution. The absence of an $f$-electron shell in $\mathrm{Y}^{3+}$ leaves it non-magnetic, whereas the half-filled $f$-shell of $\mathrm{Gd}^{3+}$ has the simplest configuration of the rare earths: a spherically symmetric $f$-shell with no orbital component $(J=S=7 / 2, L=0)$ produces a large effective moment $\mu_{\text {eff }}^{2}=g^{2} J(J+1)=\left(7.9 \mu_{\mathrm{B}}\right)^{2}$ with minimal effects from crystalline electric field anisotropy and spin-orbit coupling. The trends in $T_{\mathrm{c}}$ and $T_{\text {coh }}$ are also consistent with those found for the $\mathrm{Ce}_{1-x} \mathrm{La}_{x} \mathrm{Coln}_{5}$ series $^{15}$.

in $\mathrm{RBa}_{2} \mathrm{Cu}_{3} \mathrm{O}_{6-\delta}$ (ref. 21) owing to the large physical separation between the $\mathrm{R}$ ions and the $\mathrm{CuO}_{2}$ layers, and hence owing to negligible magnetic interaction. In $\mathrm{CeCoIn}_{5}$, evidence for evenparity pairing ${ }^{22}$ also suggests a small value of $\mathcal{g}$, given the drastic range of $D_{I}$ values (from 0.80 for $\mathrm{R}=\operatorname{Pr}$ to 15.75 for $\mathrm{R}=\mathrm{Gd}$, largest in the rare-earth series). However, in contrast to the case of the cuprates, the placement of $\mathrm{R}$ ions directly into the active pairing layer $^{18}$ of $\mathrm{CeCoIn}_{5}$ provides the first example of $T_{\mathrm{c}}$ suppression in a spin-singlet superconductor that is truly independent of $D_{J}$. Assuming the AG model applies, this places stringent bounds on both the strength of the exchange interaction involved in pairbreaking and the nature of the pairing mechanism itself.

Interestingly, this insensitivity to $D_{I}$ is mimicked in the suppression of $T_{\text {coh }}$ with rare-earth substitution, as shown in Fig. 1. The temperature $T_{\text {coh }}$ is a characteristic property of the Kondo lattice; associated with the single-ion Kondo temperature, $T_{\mathrm{K}}$ (ref. 23), and hybridization gap ${ }^{24}$, it signifies the onset of Kondo singlet formation and marks the scale where singlesite magnetic scatterers begin to dissolve into a coherent state. Interestingly, in the same way that superconductivity is destroyed when $l_{\mathrm{mfp}} \rightarrow l_{\mathrm{cr}} \approx \xi, T_{\text {coh }}$ also disappears when $l_{\mathrm{mfp}}$ approaches a characteristic coherence length $\xi_{\text {coh }} \equiv \hbar v_{\mathrm{F}} / k_{\mathrm{B}} T_{\text {coh }} \simeq 100 \AA$ (using $T_{\text {coh }}=50 \mathrm{~K}$ and $v_{\mathrm{F}} \simeq 6.5 \times 10^{-4} \mathrm{~m} \mathrm{~s}^{-1}$, where $k_{\mathrm{B}}$ and $v_{\mathrm{F}}$ are Boltzmann's constant and the Fermi velocity, respectively $)^{9}$, again with no dependence on the magnetism of the dopant ion $\mathrm{R}$. Furthermore, note that $T_{\text {coh }} \rightarrow 0$ near the $\sim 40 \%$ percolation limit
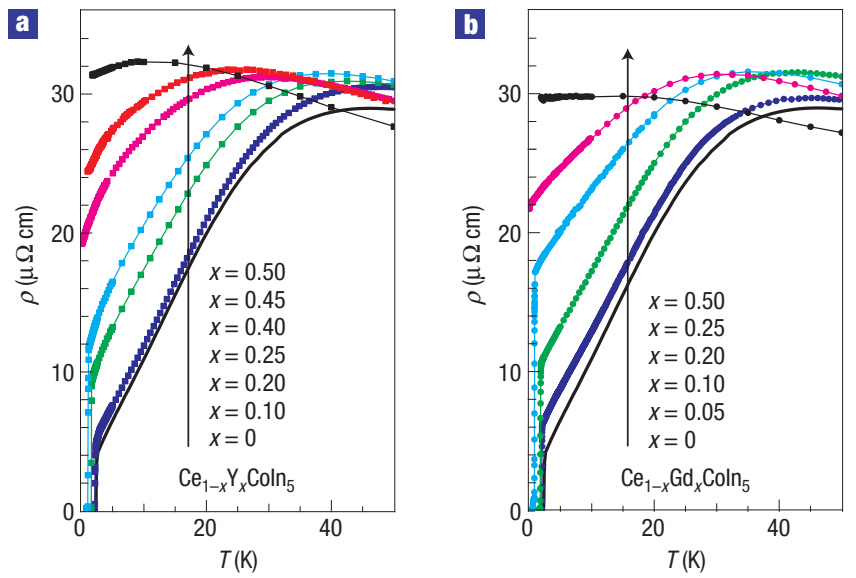

Figure 2 Comparison of electrical resistivity evolution of $\mathrm{Ce}_{1-x} \mathrm{R}_{x} \mathrm{Coln}_{5}$ with both magnetic and non-magnetic rare-earth substitution. $\mathbf{a}, \mathbf{b}$, Resistivity, $\rho$, plotted for $\mathrm{Ce}_{1-x} \mathrm{Y}_{x} \mathrm{Coln}_{5}(\mathbf{a})$ and $\mathrm{Ce}_{1-x} \mathrm{Gd}_{x} \mathrm{Coln}_{5}(\mathbf{b})$ as a function of nominal concentration of rare-earth substitution. Although both the superconducting transition and Kondo coherence temperature (maximum in $\rho(T)$ ) are suppressed at the same rate for both substitution series, the temperature dependence of $\rho$ is strongly dependent on the magnetic nature of the substituent ion: Y-doping imposes a strong downward curvature on $\rho(T)$ with increasing concentration, whereas Gd-doping elicits a negligible change in the $T$-linear resistivity present in pure $\mathrm{CeColn}_{5}$.

for a two-dimensional lattice. Together these support the notion that, regardless of its internal structure, the Ce lattice vacancy, or 'Kondo hole', is the dominant contributor to coherence destruction, leading to a universal dilution of the Kondo lattice as expected by the periodic Anderson model ${ }^{25}$. Thus, both the superconducting electron pair-breaking effect and the suppression of coherent Kondo screening proceed in a manner that is insensitive to the magnetic configuration of the dopant atom, advancing a scenario where spin-independent disorder is the dominant perturbation in both phenomena.

In contrast, the evolution of the non-Fermi-liquid electronic transport in $\mathrm{Ce}_{1-x} \mathrm{R}_{x}$ CoIn ${ }_{5}$ shows a striking sensitivity to the dopant atom's $f$-moment configuration, with $T$-linear resistivity persisting only in the presence of strong local-moment exchange. This is introduced in Fig. 2 through a direct comparison of the evolution of $\rho(T)$ as a function of both non-magnetic $\left(\mathrm{Y}^{3+}\right)$ and magnetic $\left(\mathrm{Gd}^{3+}\right)$ Ce-site substitution in $\mathrm{Ce}_{1-x} \mathrm{R}_{x} \mathrm{CoIn}_{5}$ : an increasing $\mathrm{Y}$ concentration introduces strong downward curvature in $\rho(T)$ below $T_{\text {coh }}$ (Fig. 2a), whereas $T$-linear scattering seems to be robust against magnetic Gd substitution (Fig. 2b). We further explore this duality by presenting resistivity data for several characteristic rare-earth substitutions in Fig. 3, fitting $\rho(T)$ for each between $T_{\mathrm{c}}$ and $\sim 20 \mathrm{~K}$ with a simple power law $\left(\rho=\rho_{0}+A T^{n}\right)$ and plotting $\Delta \rho=\rho-\rho_{0}$ versus $T$ to emphasize the exponent $n$, which appears as the slope on a log-log scale. As shown explicitly in the inset of Fig. 3, $n$ spans a range of sublinear values, with deviations from $T$-linear being strongest for non-magnetic substitutions.

A sub-T-linear transport scattering rate is highly anomalous, yet not unprecedented. For instance, the resistivity of the strongly correlated $f$-electron system $\mathrm{Sc}_{1-x} \mathrm{U}_{x} \mathrm{Pd}_{3}$ was indeed observed to follow the form $\rho(T)=\rho_{0}-A T^{n}$ with an exponent $n \simeq 0.5$ (ref. 26), consistent with the $n=1 / 2$ expectation of the theoretical multichannel Kondo model for $T \ll T_{\mathrm{K}}$ (ref. 9). However, the $n<1$ curvature in $\mathrm{Sc}_{1-x} \mathrm{U}_{x} \mathrm{Pd}_{3}$ is more likely due to quantum criticality associated with the suppression of spin-glass freezing to $T=0$ near $x_{\mathrm{c}} \simeq 0.3$, rather than the multichannel Kondo effect ${ }^{26}$. 
Likewise, the phenomenological trend of $n<1$ curvature in $\mathrm{Ce}_{1-x} \mathrm{R}_{x} \mathrm{CoIn}_{5}$ also hints at the proximity of a magnetic instability not unlike that found in $\mathrm{CeRhIn}_{5}$, where similar sublinear curvature is present in $\rho(T)$ above the antiferromagnetic transition at $T_{\mathrm{N}}=3.8 \mathrm{~K}$ (ref. 27). In CeRhIn , this curvature is proportional to the magnetic entropy, a reflection of the fact that magnetic correlations dominate the transport scattering process ${ }^{27}$. In $\mathrm{CeCoIn}_{5}$ the same phenomenon was found to be dependent on the proximity to a field-tuned $\mathrm{QCP}^{28}$. A connection between the two was established via resistivity measurements of the alloy series $\mathrm{CeRh}_{1-y} \mathrm{Co}_{y} \mathrm{In}_{5}$, where a crossover to sublinear behaviour in $\rho(T)$ was shown to be intimately related to the antiferromagnetic $\mathrm{QCP}^{29}$. As shown in Fig. 3, $\rho(T)$ of a single-crystal sample of $\mathrm{CeRh}_{1-y} \mathrm{Co}_{y} \mathrm{In}_{5}$ with $y=0.85$ (close to the alloy-tuned QCP) indeed follows an $n \simeq 0.5$ exponent over almost two decades in $T$ in its field-induced normal state, indicating a strong connection between $n<1$ scattering and the proximity of a QCP related to the spindensity wave instability in CeRhIn ${ }_{5}$.

In stark contrast, $\mathrm{Gd}$ substitution in $\mathrm{Ce}_{1-x} \mathrm{R}_{x} \mathrm{CoIn}_{5}$ fails to disrupt the mechanism of $T$-linear scattering: as shown in the inset of Fig. 3 , the exponent $n$ experiences an almost negligible change, decreasing at a rate at least five times slower than for non-magnetic substitutions. Because the zero-field magnetic entropy in $\mathrm{CeCoIn}_{5}$ also grows linearly with temperature above $T_{\mathrm{c}}$ (ref. 6), it is suspected that, like CeRhIn ${ }_{5}$, magnetic correlations are what shape this anomalous scattering rate. $\mathrm{In} \mathrm{Ce}_{1-x} \mathrm{Gd}_{x} \mathrm{CoIn}_{5}$, this must involve a Ruderman-Kittel-Kasuya-Yosida (RKKY)-type exchange, as demonstrated by both a linear increase with $x$ of the effective moment (up to $\mu_{\text {eff }}=7.0 \mu_{\mathrm{B}}$ at $x=1$ ) and long-range antiferromagnetic order $\left(T_{\mathrm{N}} \simeq 32 \mathrm{~K}\right.$ at $\left.x=1\right)$, which is in line with the proportionality between $T_{\mathrm{N}}$ and $D_{J}$ found in other magnetic $\mathrm{RCoIn}_{5}$ compounds $^{30}$.

But what is the underlying property of $\mathrm{Gd}^{3+}$ magnetism that is amenable to $T$-linear scattering? As shown in Fig. 3 , the curvature in $\rho(T)$ of a sample doped with $25 \% \mathrm{Er}^{3+}$-with an even larger moment $\left(\mu_{\text {eff }}=9.6 \mu_{\mathrm{B}}\right)$ than $\mathrm{Gd}^{3+}$ - surprisingly exhibits a sublinear power law $(n \simeq 0.6)$ much closer to that of the non-magnetic samples. Furthermore, samples doped with $\mathrm{Dy}^{3+}\left(\mu_{\text {eff }}=10.6 \mu_{\mathrm{B}}\right)$ exhibit intermediate behaviour, suggesting that the important parameter is not simply moment size itself, but rather involves details of the $f$-moment configuration. In particular, the wide range spanned by the de Gennes factors of $\mathrm{Gd}^{3+}, \mathrm{Dy}^{3+}$ and $\mathrm{Er}^{3+}$ (with $D_{J}$ values of $15.75,7.08$ and 2.55, respectively) is the only aspect of the magnetic configuration that follows the evolution of $n(x)$ suggested by our data set, with a phenomenological form $n \approx 1+\alpha\left(D_{J}-D_{0}\right) \rho_{0}$ where $D_{0} \simeq 18$ and $\alpha$ is a positive constant. Despite the peculiar position of $D_{I}$ in the exponent (rather than as a coefficient) of $T$, its presence highlights the important role of the spin degrees of freedom in the scattering process that gives rise to $T$-linear resistivity, promoting the notion that the 'control parameter' may indeed be the projected spin of the scattering centres.

What remains highly anomalous, and more generic, is that the relatively strong relation between $n$ and $D_{J}$ must comply with the extremely weak exchange coupling between localized $4 f$-states and conduction-band states, as demonstrated by the insensitivity of both $\Delta T_{\mathrm{c}}$ and $\Delta T_{\text {coh }}$ to the magnetic configuration of $\mathrm{R}$. This contrast provides evidence for a separation between the physics of the Kondo lattice and that of the non-Fermi-liquid transport in $\mathrm{CeCoIn}_{5}$, with the latter necessarily arising from 'incoherent' scattering processes. But how can this interaction coexist with the seemingly different longrange interactions that mediate superconductivity and resonant Kondo-lattice screening? One possibility is that the hybridization between $f$-states and conduction-electron states is incomplete,

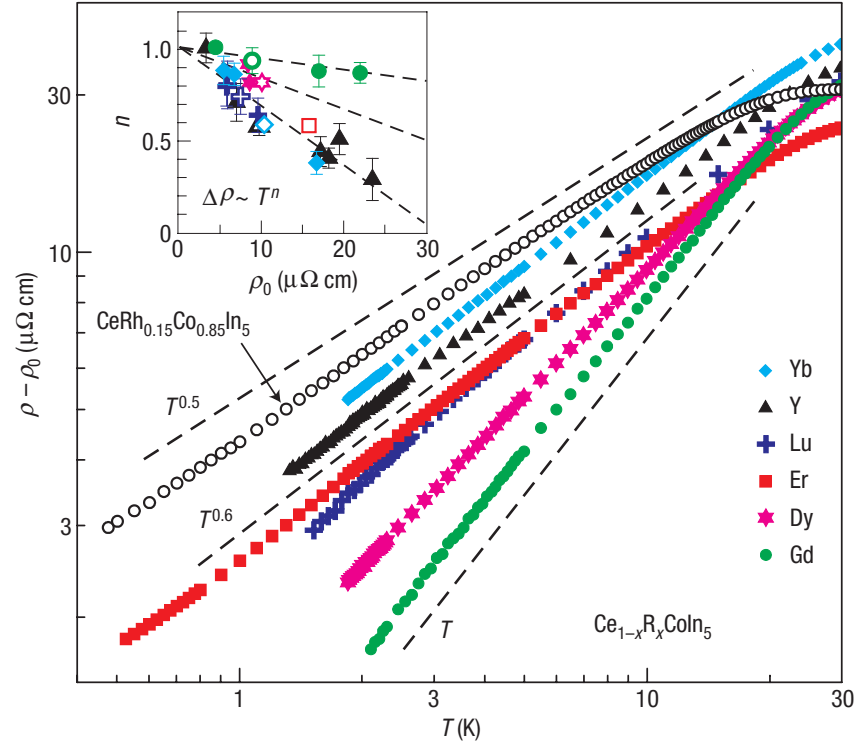

Figure 3 Effect of chemical substitution on $T$-linear resistivity power laws in CeColn $\mathbf{n}_{5}$. The filled symbols represent various rare-earth substitutions in $\mathrm{Ce}_{1-x} \mathrm{R}_{x} \mathrm{Coln}_{5}$; the open circles (shifted by $\times 2$ for clarity) represent a single-crystal sample of $\mathrm{CeRh}_{0.15} \mathrm{CO}_{0.85} \mathrm{In}_{5}$ in its field-induced normal state at $9 \mathrm{~T}$, showing the close connection between sublinear curvature in $\rho(T)$ and the proximity of a spin-density wave instability. The dashed lines are guides showing slopes for various powers of temperature. Inset: Evolution of the temperature power-law exponent $n$ (that is, in $\Delta \rho \sim T^{n}$ ) with rare-earth substitution (plotted as residual resistivity $\rho_{0}$ - see the Methods section), highlighting the isolated behaviour of Gd substitution. Whereas the large effective moment $\left(\mu_{\text {eff }}=7.9 \mu_{\mathrm{B}}\right.$ ) of $\mathrm{Gd}^{3+}$ ions in $\mathrm{Ce}_{1_{-x}} \mathrm{R}_{x} \mathrm{Coln}_{5}$ sets it apart from its non-magnetic counterparts, the sublinear exponent observed for $\mathrm{Er}^{3+}$ substitution (red square)—with $\mu_{\text {eff }}=9.6 \mu_{\mathrm{B}}$-rules out a simple correlation between the moment size and the sublinear power-law exponent, suggesting the importance of the spin configuration of the rare-earth ions and de Gennes factor scaling. The error bars reflect estimates of uncertainty in $n$ on the basis of the temperature range and number of data points used in nonlinear least-squares fits of $\rho(T)$. (The open symbols denote concentrations used in the main figure.)

leaving a fraction of incoherent scatterers that conspire to cause such a dichotomy. Evidence for such two-fluid behaviour does indeed take form in $\mathrm{CeCoIn}_{5}$, where an 'incoherent' fraction of Kondo moments was shown to survive down to $T_{c}$ (ref. 31). Another scenario is of a more profound nature: recent evidence for (1) a group of conduction electrons that remains unpaired in the $T \rightarrow 0$ limit $^{32}$ and (2) a direction-dependent violation of the Wiedemann-Franz law ${ }^{14}$ points to a decoupled character of conduction electrons in $\mathrm{CeCoIn}_{5}$, suggesting that the separation between the mechanisms behind the coherent properties of $\mathrm{CeCoIn}_{5}$ and its $T$-linear resistivity is of a very fundamental nature.

\section{METHODS}

Single-crystal platelets of $\mathrm{Ce}_{1-x} \mathrm{R}_{x} \mathrm{CoIn}_{5}$ (including $\mathrm{R}=\mathrm{Y}, \mathrm{Pr}, \mathrm{Gd}, \mathrm{Dy}, \mathrm{Er}, \mathrm{Yb}$ and $\mathrm{Lu}$ ) were grown by the self-flux method ${ }^{6}$. Samples for measurements of electrical resistivity were prepared with typical dimensions $\sim 2 \times 0.5 \times 0.2 \mathrm{~mm}$ and measured with an a.c. resistance bridge by applying $\sim 0.1 \mathrm{~mA}$ excitation current, directed parallel to the basal plane of the tetragonal crystal structure. The data in Figs 1 and 2 are plotted as a function of residual resistivity to eliminate the uncertainty in nominal concentration values. However, note that $\rho_{0} \sim x$ to within error as found previously ${ }^{15,32}$. The d.c. magnetization was 
measured using a superconducting quantum interference device magnetometer in a $50 \mathrm{mT}$ field, and analysed using standard Curie-Weiss fits to data between approximately 25 and $300 \mathrm{~K}$ to extract effective moments for the magnetic $\mathrm{Ce}_{1-x} \mathrm{R}_{x} \mathrm{CoIn}_{5}$ series.

\section{Received 16 May 2007; accepted 3 August 2007; published 9 September 2007.}

\section{References}

1. Seaman, C. L. et al. Evidence for non-Fermi liquid behavior in the Kondo alloy $\mathrm{Y}_{1-x} \mathrm{U}_{x} \mathrm{Pd}_{3}$. Phys. Rev Lett. 67, 2882-2885 (1991).

2. Löhneysen, H. v. et al. Non-Fermi-liquid behavior in a heavy-fermion alloy at a magnetic instability. Phys. Rev. Lett. 72, 3262-3265 (1994).

3. Trovarelli, O. et al. $\mathrm{YbRh}_{2} \mathrm{Si}_{2}$ : Pronounced non-Fermi-liquid effects above a low-lying magnetic phase transition. Phys. Rev. Lett. 85, 626-629 (2000).

4. Holmes, A. T., Jaccard, D. \& Miyake, K. Signatures of valence fluctuations in $\mathrm{CeCu}_{2} \mathrm{Si}_{2}$ under high pressure. Phys. Rev. B 69, 024508 (2004).

5. Martin, S., Fiory, A. T., Fleming, R. M., Schneemeyer, L. F. \& Waszczak, J. V. Normal-state transport properties of $\mathrm{Bi}_{2+x} \mathrm{Sr}_{2-y} \mathrm{CuO}_{6+\delta}$ crystals. Phys. Rev. B 41, 846-849 (1990).

6. Petrovic, C. et al. Heavy-fermion superconductivity in $\mathrm{CeCoIn}_{5}$ at 2.3 K. J. Phys. Condens. Matter 13 L337-L342 (2001).

7. Coleman, P. \& Schofield, A. J. Quantum criticality. Nature 433, 226-229 (2005).

8. Varma, C., Littlewood, P. B., Schmitt-Rink, S., Abrahams, E. \& Ruckenstein, A. E. Phenomenology of the normal state of $\mathrm{Cu}-\mathrm{O}$ high-temperature superconductors. Phys. Rev. Lett. 63, 1996-1999 (1989)

9. Cox, D. L. \& Jarrell, M. J. The two-channel Kondo route to non-Fermi-liquid metals. J. Phys. Condens. Matter 8, 9825-9853 (1996).

10. Rosch, A., Schröder, A., Stockert, O. \& Löhneysen, H. v. Mechanism for the non-Fermi-liquid behavior in $\mathrm{CeCu}_{6-x} \mathrm{Au}_{x}$. Phys. Rev. Lett. 79, 159-162 (1997).

11. Mackenzie, A. P., Julian, S. R., Sinclair, D. C. \& Lin, C. T. Normal-state magnetotransport in superconducting $\mathrm{Tl}_{2} \mathrm{Ba}_{2} \mathrm{CuO}_{6+\delta}$ to millikelvin temperatures. Phys. Rev. B 53, 5848-5855 (1996).

12. Nakajima, Y. et al. Normal-state Hall angle and magnetoresistance in quasi-2D heavy fermion $\mathrm{CeCoIn}_{5}$ near a quantum critical point. J. Phys. Soc. Japan 73, 5-8 (2004).

13. Phillips, P. \& Chamon, C. Breakdown of one-parameter scaling in quantum critical scenarios fo high-temperature copper-oxide superconductors. Phys. Rev. Lett. 95, 107002 (2005).

14. Tanatar, M. A., Paglione, J., Petrovic, C. \& Taillefer, L. Anisotropic violation of the Wiedemann-Fran law at a quantum critical point. Science 316, 1320-1322 (2007).

15. Petrovic, C., Bud'ko, S. L., Kogan, V. G. \& Canfield, P. C. Effects of La substitution on the superconducting state of CeCoIn ${ }_{5}$. Phys. Rev. B 66, 054534 (2002).

16. Kasahara, Y. et al. Anomalous quasiparticle transport in the superconducting state of $\mathrm{CeCoIn}_{5}$. Phys Rev. B 72, 214515 (2005)

17. Bauer, E. D. et al. Thermodynamic and transport investigation of CeCoIn ${ }_{5-x} \operatorname{Sn}_{x}$. Phys. Rev. B 73 245109 (2006).
18. Daniel, M. et al. Perturbing the superconducting planes in CeCoIn ${ }_{5}$ by Sn substitution. Phys. Rev. Lett. 95, 016406 (2005).

19. Maple, M. B. \& Fischer, Ø. (eds) Superconductivity in Ternary Compounds Vols 1 \& 2 (Springer, Berlin, 1982).

20. Dalichaouch, Y. et al. Impurity scattering and triplet superconductivity in UPt $\mathrm{UP}_{3}$. Phys. Rev. Lett. 75, 3938-3941 (1995).

21. Hor, P. H. et al. Superconductivity above $90 \mathrm{~K}$ in the square-planar compound system $\mathrm{ABa}_{2} \mathrm{Cu}_{3} \mathrm{O}_{6+x}$ with A = Y, La, Nd, Sm, Eu, Gd, Ho, Er and Lu. Phys. Rev. Lett. 58, 1891-1894 (1987).

22. Higemoto, W. et al. $\mu$ SR studies on heavy fermion superconductors CeIrIn ${ }_{5}$ and CeCoIn $\mathrm{C}_{5}$. J. Phys. Soc. Japan 71, 1023-1026 (2002).

23. Burdin, S., Georges, A. \& Grempel, D. R. Coherence scale of the Kondo lattice. Phys. Rev. Lett. 85, $1048-1051(2000)$

24. Dordevic, S. V., Basov, D. N., Dilley, N. R., Bauer, E. D. \& Maple, M. B. Hybridization gap in heavy fermion compounds. Phys. Rev. Lett. 86, 684-687 (2001).

25. Wermbter, S., Sabel, K. \& Czycholl, G. Electrical resistivity of heavy-fermion systems with nonmagnetic impurities. Phys. Rev. B 53, 2528-2536 (1996).

26. Dickey, R. P. et al. Interplay between magnetism and non-Fermi-liquid behavior in $\mathrm{Sc}_{1-x} \mathrm{U}_{x} \mathrm{Pd}_{3}$. Phys. Rev. B 68, 104404 (2003).

27. Paglione, J. et al. Heat transport as a probe of electron scattering by spin fluctuations: the case of antiferromagnetic CeRhIn 5 . Phys. Rev. Lett. 94, 216602 (2005)

28. Paglione, J. et al. Nonvanishing energy scales at the quantum critical point of CeCoIn 5 . Phys. Rev. Lett. 97, 106606 (2006)

29. Jeffries, J. R. et al. Superconductivity and non-Fermi liquid behavior near antiferromagnetic quantum critical points in $\mathrm{CeRh}_{1-y} \mathrm{Co}_{y} \mathrm{In}_{5}$. Phys. Rev. B 72, 024551 (2005).

30. Isikawa, Y., Kato, D., Mitsuda, A., Mizushima, T. \& Kuwai, T. Magnetic properties of single crystals of $\mathrm{RCoIn}_{5}(\mathrm{R}=\mathrm{Tb}, \mathrm{Dy}, \mathrm{Ho}, \mathrm{Er}, \mathrm{Yb})$. J. Magn. Magn. Mater. 272, 635-636 (2004).

31. Nakatsuji, S., Pines, D. \& Fisk, Z. Two fluid description of the Kondo lattice. Phys. Rev. Lett. 92 016401 (2004).

32. Tanatar, M. A. et al. Unpaired electrons in the heavy-fermion superconductor CeCoIn ${ }_{5}$. Phys. Rev. Lett. 95, 067002 (2005).

\section{Acknowledgements}

The authors acknowledge B. Coqblin, P. Coleman, C. Pépin and C. Petrovic for useful discussions and P. Johnson for assistance in sample preparation. Crystal growth and characterization was sponsored by the US Department of Energy (DOE) under research grant DE-FG02-04ER46105, and low-temperature experiments by the National Science Foundation under grant No. 0335173. J.P. acknowledges support from a NSERC Canada postdoctoral fellowship.

Correspondence and requests for materials should be addressed to J.P.

Competing financial interests

The authors declare no competing financial interests.

Reprints and permission information is available online at http://npg.nature.com/reprintsandpermissions/ 